This item was submitted to Loughborough's Research Repository by the author.

Items in Figshare are protected by copyright, with all rights reserved, unless otherwise indicated.

\title{
Bayesian inference for vehicle speed and vehicle length using dual-loop detector data
}

PLEASE CITE THE PUBLISHED VERSION

http://www.sciencedirect.com/science/article/pii/S0191261509000782

PUBLISHER

(C) Elsevier

VERSION

AM (Accepted Manuscript)

LICENCE

CC BY-NC-ND 4.0

\section{REPOSITORY RECORD}

Li, Baibing. 2019. "Bayesian Inference for Vehicle Speed and Vehicle Length Using Dual-loop Detector Data". figshare. https://hdl.handle.net/2134/9163. 
This item was submitted to Loughborough's Institutional Repository (https://dspace.lboro.ac.uk/) by the author and is made available under the following Creative Commons Licence conditions.

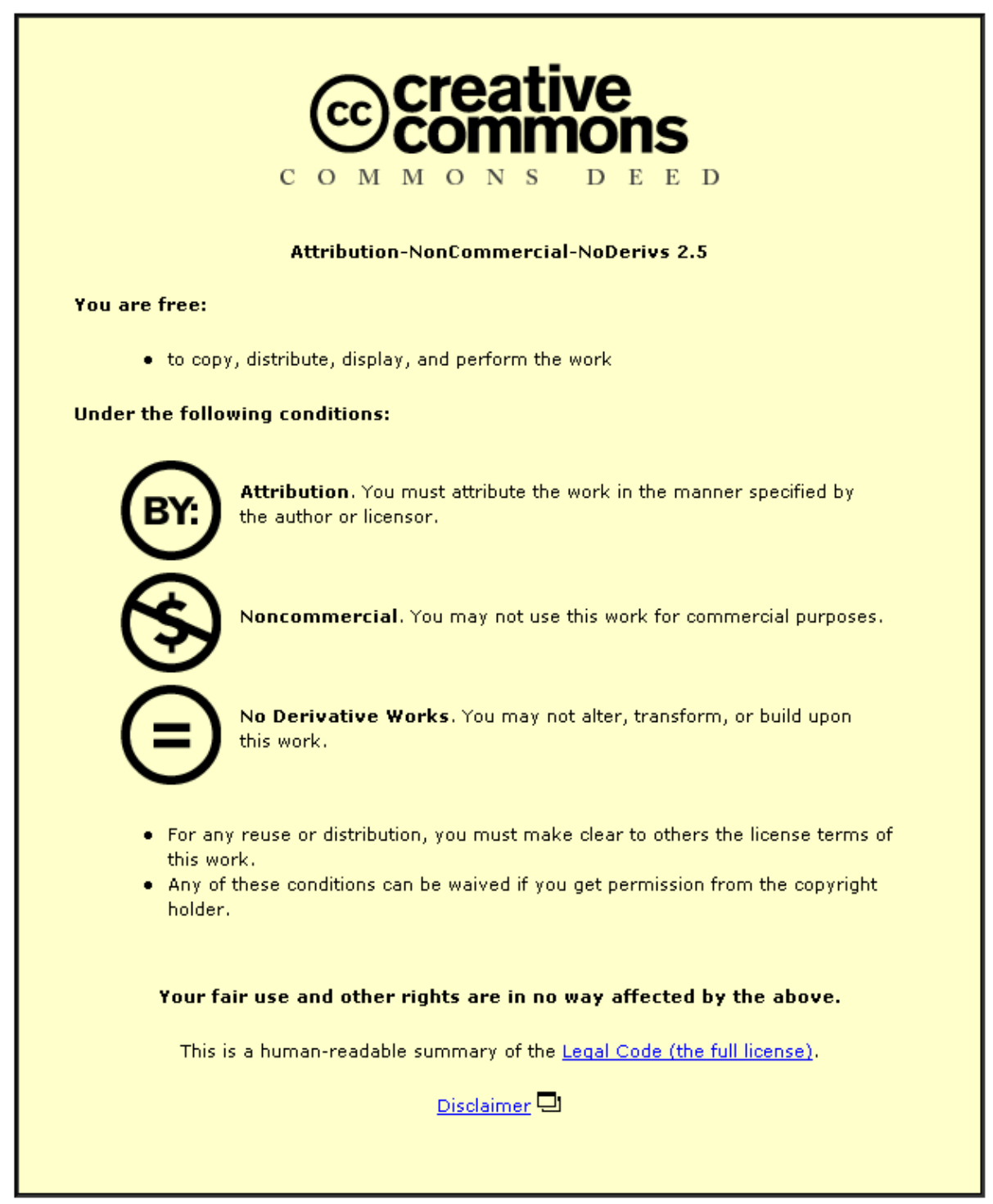

For the full text of this licence, please go to: http://creativecommons.org/licenses/by-nc-nd/2.5/ 


\title{
Bayesian inference for vehicle speed and vehicle length \\ using dual-loop detector data
}

\author{
Baibing Li \\ Business School \\ Loughborough University \\ Loughborough LE11 3TU, United Kingdom \\ (email: b.li2@lboro.ac.uk)
}

\begin{abstract}
A dual-loop detector consists of two connected single-loop detectors placed several feet apart. Compared with a single-loop detector, it is able to provide more useful information on traffic flow with a higher precision. In this paper we investigate statistical inference for vehicle speed and vehicle length using dual-loop detector data. A Bayesian analysis is performed to combine current observations on traffic flow with prior knowledge, which results in a set of simple formulas for the online estimation of both vehicle speed and vehicle length. As a by-product, vehicle classification is also investigated on the basis of posterior classification probabilities. The computational overhead of updating the estimates is kept to a minimum when new information on traffic flow becomes available. The method is illustrated using real traffic data.
\end{abstract}

Keywords: Bayesian analysis; Dual-loop detector; Vehicle classification; Vehicle length;

Vehicle speed 


\section{Introduction}

Dual-loop detectors play a crucial role in advanced traffic management systems. They provide estimates of fundamental parameters of traffic flow such as vehicle speed and length. It is therefore essential that the information from a dual-loop detector is analysed as accurately as possible with a minimum computational cost. The aim of this paper is to draw statistical inference for vehicle speed and length using dual-loop detector data.

In recent years, considerable attention has been paid to single-loop detectors. The data from a single-loop detector are provided at an aggregated level, where traffic volume and occupancy are recorded during pre-determined time intervals (each is typically 20 to 30 seconds long). Based on the information from a single-loop detector, space-mean-speed can be calculated under the assumption that effective vehicle length is constant and exogenously available.

In the classical estimation method for single-loop data, the calculated space-mean-speed is used as an estimate of current vehicle speed (see, e.g., Kurkjian et al. 1980; Hazelton 2004). A serious drawback of the classical method is that only one piece of data, i.e., the current space-mean-speed observation, is used for estimation, and thus the quality of the estimator is poor. This classical approach has been greatly improved in the last decade via a pooling of the information obtained in previous time periods. For instance, Dailey (1999) investigated using the Kalman filter to estimate vehicle speed. Through some approximations he derived a linear observation equation and then applied the Kalman filter to obtain a set of recursive formulas. The approach of using the Kalman filter has also been investigated by Ye et al. (2006) and Bickel et al. (2007). Hazelton (2004) has carried out a full Bayesian analysis in which a Markov chain Monte Carlo (MCMC) approach is incorporated to draw statistical inference. Recently Li (2009) has developed a recursive method for fast estimation of vehicle speed, where the current estimate is updated as a weighted harmonic average of the current 
space-mean-speed and the previous estimate. It is faster than the MCMC approach of Hazelton (2004) and avoids the linearization made in Dailey's method.

A dual-loop detector consists of two connected single loops, the upstream loop and the downstream loop. Unlike single-loop detectors however, observations from a dual-loop detector are available on a vehicle-by-vehicle basis. Consequently, a dual-loop detector can provide much more accurate information on traffic flow, such as vehicle speed, vehicle length, and truck volume. However, the currently used estimation method for dual-loop data suffers from the same problem as does the classical method for single-loop detectors: only the current observations are used for estimation. The resulting estimates of vehicle speed and length are very noisy. In addition, no measure of the quality of the estimates, such as standard errors, is provided.

It is surprising that relatively little research has been done on dual-loop detectors. In recent years there have been only a few studies, mainly focusing on the data validation of dual-loop detectors. The signals received by a dual-loop detector consist of a sequence of 0 and 1 digits. In practice, a positive or negative single-digit false will sometimes occur, i.e., a digit of 0 (or 1) is mistakenly input as 1 (or 0). Zhang et al. (2006) developed a robust algorithm to eliminate such erroneous inputs that included a noise filter and a postprocessor to screen out noise, and a matching scheme to pair up on-time pulses. They also considered various checks to test the validity of the data for the calculation of vehicle speed and length. Cheevarunothai et al. (2006) investigated how to enhance the reliability of dual-loop data via removing the sensitivity discrepancy between the two single loops and adjusting their sensitivities to the appropriate level. Coifman and Dhoorjaty (2004) proposed eight detector validation tests for freeway surveillance, where the extracted vehicle information was compared with corresponding pre-set constant thresholds to identify erroneous loop data. The 
signal validation and improvement in these studies have laid a solid foundation for subsequent data analysis.

In this paper we extend the estimation method for single-loop data developed by Li (2009) to the scenario of dual-loop problems where statistical inference for both vehicle speed and vehicle length is drawn. We perform Bayesian analysis to pool the information obtained previously with the current observations to improve the estimates of vehicle speed and length. As in Li (2009) for single-loop detectors, the Bayesian analysis results in a set of simple formulas that are analytically pleasing. As a by-product, vehicle classification is also investigated by comparing the posterior classification probabilities that a vehicle falls into different vehicle classes. The developed method incurs a minimum computational cost, and can easily be implemented in practice.

This paper is structured as follows. Section 2 is devoted to the problem formulation. A statistical model for dual-loop data is developed in Section 3. Then Bayesian analysis is performed to estimate the vehicle speed and length in Section 4. To illustrate the proposed method, numerical examples are examined in Section 5. Finally, concluding remarks are offered in Section 6. All proofs are given in the Appendix.

\section{Problem formulation}

\subsection{Notation}

Consider a dual-loop detector that consists of two connected single-loops, an upstream loop and a downstream loop, as illustrated in Figure 1. In practice, the sensitivity region of a single loop may differ from the area defined by its physical boundary (Hazelton, 2004; Cheevarunothai et al., 2006). Let

- $\quad M$ be the distance between the leading edge of the upstream loop's sensitivity region and the leading edge of the downstream loop's sensitivity region; 
- $L_{d}$ be the length of the sensitivity region of the downstream loop.

Note that if the two single loops have sensitivity regions of equal size, then $M$ is equal to the distance between the leading edge of the upstream loop's physical boundary and the leading edge of the downstream loop's physical boundary (Cheevarunothai et al., 2006).

When a vehicle traverses the dual-loop detector, the controller of the detector normally records four transitions, i.e., turn-on and turn-off times at each of the single loops. They are represented as a vector of observations, $T=\left[t_{\text {uon }}, t_{\text {uoff }}, t_{d o n}, t_{d o f f}\right]^{T}$, where

- $t_{\text {uon }}$ is the time when a vehicle enters the leading edge of the upstream loop's sensitivity region;

- $t_{\text {uoff }}$ is the time when a vehicle leaves the rear edge of the upstream loop's sensitivity region;

- $t_{d o n}$ is the time when a vehicle enters the leading edge of the downstream loop's sensitivity region;

- $t_{\text {doff }}$ is the time when a vehicle leaves the rear edge of the downstream loop's sensitivity region.

In this paper two parameters of interest, vehicle speed and vehicle length, denoted as $v$ and $L$ respectively, are to be estimated. It is assumed throughout this paper that when a vehicle traverses the two loops of the detector, its speed $v$ maintains a constant level. This assumption is reasonable because the distance between the two loops is very short. For different vehicles, however, the vehicle speed may differ.

(Figure 1 is about here)

Figure 1. An illustration of a dual-loop detector. 


\subsection{The classical estimation method}

In the classical estimation method, vehicle speed is calculated as follows (see, e.g., Cheevarunothai et al., 2006; Zhang et al. 2006):

$$
s=M /\left(t_{d o n}-t_{u o n}\right) .
$$

Once an estimate of vehicle speed has been obtained, the effective vehicle length associated with the downstream loop can be estimated as

$$
D=s\left(t_{\text {doff }}-t_{d o n}\right)
$$

Based on equation (2-2), an estimate of vehicle length can be obtained: $D-L_{d}$.

From the above equations it can be seen that in the classical method the speed of a vehicle is assumed to be constant when the vehicle travels through the dual loop detector.

We also note that for vehicle speed and effective vehicle length, the classical method provides point estimates only, and thus no measure for the quality of the estimates is available from the method. In addition, the estimates are based solely on the current observations. Therefore, better estimates could be obtained once the information collected previously was incorporated.

\section{A statistical model for dual-loop detector data}

\subsection{The model}

Let $L_{e}=L_{d}+L$ denote the effective vehicle length associated with the downstream loop. Since $L_{d}$ is a constant, statistical inference can be drawn for the parameter of effective vehicle length $L_{e}$ rather than the vehicle length $L$ itself.

Let $x=t_{d o n}-t_{\text {uon }}$ be the time required by a vehicle to travel the distance $M$ from the leading edge of the upstream loop's sensitivity region to the leading edge of the downstream 
loop's sensitivity region. Likewise, let $y=t_{d o f f}-t_{d o n}$ be the time required by a vehicle to travel the distance $L_{e}$.

We shall develop a statistical model for the paired data $x$ and $y$ measured on the same vehicle for statistical inference about the unknown parameters $v$ and $L_{e}$. We first note that for a vehicle traversing the dual-loop detector, the paired time measurements $x$ and $y$ are affected by the driver's behavior, and thus they are not independent of each other. Note, however, when a multiplicative model is assumed for the time measurements $x$ and $y$ which are affected by the random effect of a driver's behavior, the random effect will be eliminated when the ratio of $y$ to $x$, i.e. $r=y / x$, is calculated. Likewise, when a common vehicle speed $v$ is assumed for the same vehicle passing through the upstream loop and downstream loop, it is also expected that the ratio $r=y / x$ depends on the ratio of the two distances $L_{e}$ and $M$ only, where the common speed $v$ is cancelled out.

The ratio $r=y / x$ thus provides the required information for drawing statistical inference about the unknown effective vehicle length $L_{e}$ without involving the speed parameter $v$. On the other hand, statistical inference for vehicle speed $v$ can be drawn using the information contained in time measurement $x$ which does not involve the other unknown parameter $L_{e}$. In doing so, the estimation problem of the two unknown parameters $v$ and $L_{e}$ is disentangled.

Now we begin to specify a statistical model for the measurements $x$ and $y$. First, following Polus (1979) and Li (2009), the marginal distribution of $x$ is assumed to be a gamma distribution:

$$
x \mid v \sim \Gamma\left(\alpha, \alpha v M^{-1}\right)
$$


with a mean of $M / v$ and a variance of $(M / v)^{2} \alpha^{-1}$, where $\alpha$ is a diffusion parameter. $\Gamma(a, b)$ is a gamma distribution having probability density function $g(x ; a, b)=\left\{b^{a} / \Gamma(a)\right\} x^{a-1} \exp (-b x)$ with $\Gamma(a)$ the gamma function.

On the basis of the foregoing discussion, the conditional distribution of $r=y / x$ given $x$, $p\left(r \mid x, L_{e}\right)$, will be specified to be a distribution with a mean equal to $L_{e} / M$ without involving the speed parameter $v$. Ideally the resulting marginal distribution of $y$ will approximately have a gamma distribution due to the empirical results in Polus (1979).

In the recent decades many multivariate gamma distributions have been developed (see Kotz et al. (2000), Chapter 48, for a comprehensive review) but most of them are far too complicated for the online estimation problem of vehicle speed and vehicle length. The bivariate gamma distribution developed by McKay, however, has some very nice features and is analytical tractable. The two marginal distributions of McKay's bivariate gamma distribution also are gamma. The conditional distribution of $y$ given $x$ has a scaled beta distribution, $(y / x) \mid x \sim \operatorname{beta}(a, b)$, with the following density (Kotz et al., 2000, pp 432):

$$
p(y \mid x)=\frac{\Gamma(a+b)}{\Gamma(a) \Gamma(b)}\left(\frac{y}{x}\right)^{a-1}\left(1-\frac{y}{x}\right)^{b-1} x^{-1}
$$

with range $y \in(0, x)$.

For the problem considered here, it is clear that the range of the random variables of the above conditional distribution is too restrictive. Since effective vehicle length is normally over 16 feet, i.e. $L_{e}>M=16$ feet, it is expected that $y>x$ for most of vehicles. Assuming a scaled inverse beta distribution $(y / x) \mid x \sim \operatorname{inv}-\operatorname{beta}(a, b)$ with a range of $y \in(x,+\infty)$ does not completely solve this difficulty because for real traffic data, there also exist a small proportion of vehicles with $y \leq x$. 
To address this issue, the inverse beta distribution is replaced by a more flexible inverse gamma distribution in this paper, $(y / x) \mid x \sim \operatorname{inv} \Gamma(a, b)$, so that $y$ can take any non-negative values, where a random variable $\xi$ follows an inverse gamma distribution $\operatorname{inv} \Gamma(a, b)$ if $\xi^{-1} \sim \Gamma(a, b)$. Specifically, we consider the following model for the time measurement $y$ given $x$ :

$$
y \mid\left(x, L_{e}\right) \sim \operatorname{inv} \Gamma\left(\beta,(\beta-1) L_{e} x / M\right) \quad \text { with } \beta>2 .
$$

By defining the ratio $r=y / x$, the distribution (3-2) can be rewritten as:

$$
r \mid\left(x, L_{e}\right) \sim \operatorname{inv} \Gamma\left(\beta,(\beta-1)\left(L_{e} / M\right)\right) .
$$

As required, the distribution in (3-3) has a mean equal to the ratio of the distances $L_{e} / M$. The coefficient of variation $(\beta-2)^{-1 / 2}$ of the distribution (3-3) is solely determined by the diffusion parameter $\beta$ which provides flexibility to fit data.

Combining (3-1) and (3-2), the joint distribution of $x$ and $y$ is given by

$$
\begin{aligned}
& p\left(x, y \mid v, L_{e}\right)=p(x \mid v) p\left(y \mid x, L_{e}\right) . \\
& =c_{1} y^{-(\beta+1)} x^{\alpha+\beta-1} \exp \left\{-\left[\alpha v / M+(\beta-1) L_{e} /(M y)\right] x\right\}
\end{aligned}
$$

where $c_{1}=(\alpha v / M)^{\alpha}\left\{(\beta-1) L_{e} / M\right\}^{\beta}\{\Gamma(\alpha) \Gamma(\beta)\}^{-1}$ is a constant. In the next subsection we shall show that the joint distribution (3-4) has some desirable properties.

\subsection{Properties of the distribution of the time measurements}

On the basis of the joint distribution (3-4), the marginal distribution of $y$ can be derived.

Theorem 1. The marginal distribution of $y$ is a scaled $F$ distribution with degrees of freedom $2 \alpha$ and $2 \beta$ respectively, i.e. $\left\{v \beta /\left(L_{e}(\beta-1)\right)\right\} y \sim F(2 \alpha, 2 \beta)$, with a mean of $L_{e} / v$ and a 
variance of $\left(L_{e} / v\right)^{2} /\left(\alpha \rho^{2}\right)$, where $\rho=\{(\beta-2) /(\alpha+\beta-1)\}^{1 / 2}>0$ is the correlation coefficient for $x$ and $y$.

Overall, the correlation for $x$ and $y$ is strong (or weak) if $\beta$ is large (or small), and/or if $\alpha$ is small (or large). The following limiting cases are observed: (i) $\rho \rightarrow 1$ as $\beta \rightarrow+\infty$ but $\alpha$ is fixed; (ii) $\rho \rightarrow 0$ as $\beta \rightarrow 2$ but $\alpha$ is fixed; (iii) $\rho \rightarrow 0$ as $\alpha \rightarrow+\infty$ but $\beta$ is fixed; (iv) $\rho \rightarrow\{(\beta-2) /(\beta-1)\}^{1 / 2} \approx 1$ as $\alpha \rightarrow 0$ and $\beta$ is not small.

When both $\alpha$ and $\beta$ are large, an approximate distribution of $y$ can be obtained. Specifically, consider a normalized variable $z=\left(v / L_{e}\right) y$ having the following probability density function

$$
f(z ; \alpha, \beta)=\{\Gamma(\alpha+\beta) /(\Gamma(\alpha) \Gamma(\beta))\} \alpha^{\alpha}(\beta-1)^{\beta} z^{\alpha-1} /\{(\beta-1)+\alpha z\}^{\alpha+\beta} .
$$

We have the following result.

Lemma 1. For the distribution $f(z ; \alpha, \beta)$ in (3-5) and the gamma distribution $g(z ; \phi, \phi)=\left\{\phi^{\phi} / \Gamma(\phi)\right\} z^{\phi-1} \exp (-\phi z)$ with $\phi=\alpha \rho^{2}$, (i) their means and variances are equal; (ii) the ratio of the $r$ th moments about zero $(r>2)$ of the two distributions is of order $1+O(1 / \alpha)+O(1 / \beta)$.

Lemma 1 indicates that when $\alpha$ and $\beta$ are large, the marginal distribution of $y$ given by Theorem 1 can be well approximated by the gamma distribution $\Gamma\left(\phi, \phi v / L_{e}\right)$. This is desirable since according to the existing empirical results (see, e.g., Polus, 1979), the time required for a vehicle to travel a fixed distance approximately follows a gamma distribution.

\section{Bayesian inference}


In this section, we perform Bayesian analysis to develop a method for the estimation of vehicle speed and effective vehicle length. Suppose that a vehicle has just traversed the dualloop detector and a piece of information on this vehicle, $T=\left[t_{\text {uon }}, t_{\text {uoff }}, t_{\text {don }}, t_{\text {doff }}\right]^{T}$, is available. Let $x=t_{d o n}-t_{\text {uon }}$ and $y=t_{d o f f}-t_{d o n}$. For simplicity, we first base the statistical analysis solely on the paired data $(x, y)$. The developed method will then be extended in Section 4.6 where the time measurements $t_{\text {doff }}-t_{\text {uoff }}$ and $t_{\text {uoff }}-t_{\text {uon }}$ are also incorporated into the analysis.

\subsection{Prior distributions}

To perform Bayesian analysis, prior distributions for the parameters of effective vehicle length $L_{e}$ and vehicle speed $v$ need to be specified first.

It has long been known that the distribution of effective vehicle lengths is a mixture distribution with multiple components. For instance, a multimodal distribution was suggested in the empirical analysis by Hazelton (2004), where each of the modes was linked to a vehicle class, including (a) cars; (b) vans and small lorries; and (c) large lorries and road trains. In this paper, a parametric model is considered and the prior distribution of $L_{e}$ is specified as the following mixture of $Q$ gamma distributions:

$$
p\left(L_{e}\right)=\sum_{i=1}^{Q} q_{i} g\left(L_{e} ; b_{i}, b_{i} \lambda_{i}^{-1}\right),
$$

where the weight $q_{i}$ is the prior classification probability of vehicle class $i$, i.e. $q_{i}=\operatorname{Pr}\{$ vehicle $\in$ class $i\}$. The weights satisfy $\quad q_{i}>0 \quad$ and $\sum_{i=1}^{Q} q_{i}=1$. The density $g\left(L_{e} ; b_{i}, b_{i} \lambda_{i}^{-1}\right)$ of each component in (4-1) is the prior distribution of $L_{e}$ for each vehicle class, i.e. $p\left(L_{e} \mid\right.$ class $\left.i\right)=g\left(L_{e} ; b_{i}, b_{i} \lambda_{i}^{-1}\right)$. For vehicles falling into a category characterized 
by each component $g\left(L_{e} ; b_{i}, b_{i} \lambda_{i}^{-1}\right)$, the prior mean of effective vehicle length is $\lambda_{i}$ and hyper-parameter $b_{i}$ is associated with the prior precision.

The prior distribution of $L_{e}$ in (4-1) needs to be estimated from the collected exogenous data of vehicle lengths. In practice usually the sample size is large and the precision of the estimation is high. The EM algorithm can be used to estimate the hyper-parameters in (4-1). See McLachlan (1996) for details.

Next, we turn to specify a prior for vehicle speed $v$. Following $\operatorname{Li}(2009)$, the prior for $v$ is specified as a gamma distribution:

$$
v \sim \Gamma\left(a, a \mu^{-1}\right),
$$

where the prior mean is equal to $\mu$ and the prior standard deviation is equal to $\mu / a^{1 / 2}$. The prior mean $\mu$ represents an estimate of speed parameter $v$ obtained a priori. The hyperparameter $a$ reflects the accuracy of the prior information about $v$.

Usually a non-informative prior of $v$ is incorporated for the first vehicle under investigation, $v \sim \Gamma\left(a_{0}, a_{0} \mu_{0}^{-1}\right)$, where $\mu_{0}$ can be taken as any reasonable value between 0 to 100 and $a_{0}$ is taken sufficiently small, say $10^{-6}$, resulting in a sufficiently large prior variance.

Following Dailey (1999), and Wang and Nihan (2000), we assume that the parameters of interest, $v$ and $L_{e}$, are independent of each other a priori:

$$
p\left(v, L_{e}\right)=p(v) p\left(L_{e}\right) .
$$

\subsection{Bayesian analysis}

Now we apply Bayes' rule to combine the prior (4-3) with the likelihood (3-4). The derived posterior distribution is given by: 


$$
p\left(v, L_{e} \mid x, y\right) \propto p\left(x, y \mid v, L_{e}\right) p\left(v, L_{e}\right)=\{p(x \mid v) p(v)\}\left\{p\left(y \mid x, L_{e}\right) p\left(L_{e}\right)\right\} .
$$

Note that the joint posterior distribution of $v$ and $L_{e}$ is disentangled so that the posterior analysis can be carried out separately.

We first focus on statistical inference for the speed parameter $v$. According to Li (2009), the posterior $p(x \mid v) p(v)$ also is a gamma distribution given by $\Gamma\left(a+\alpha, a \mu^{-1}+\alpha s^{-1}\right)$, where $s=M / x$ is the current speed observation calculated using equation (2-1). The posterior mean of $v$ can be obtained, $E(v \mid x)=\left\{\theta \mu^{-1}+(1-\theta) s^{-1}\right\}^{-1}$, where weight $\theta$ is given by $\theta=a /(a+\alpha)$. Denote the posterior mean $E(v \mid x)$ as $\mu^{*}$ which is used in this paper to estimate the current vehicle speed. It is a weighted harmonic average of the previous estimate $\mu$ and the currently calculated speed observation $s$ :

$$
\mu^{*}=\left\{\theta \mu^{-1}+(1-\theta) s^{-1}\right\}^{-1}
$$

Let $a^{*}=a+\alpha$. Then the posterior distribution of $v$ can be rewritten as

$$
v \mid x \sim \Gamma\left(a^{*}, a^{*} \mu^{*-1}\right) .
$$

The posterior variance is $\operatorname{var}(v \mid x)=\mu^{* 2} / a^{*}$. A $95 \%$ credible interval for $v$ is given by

$$
\left(\mu^{*} \chi_{0.975}^{2}\left(2 a^{*}\right) /\left(2 a^{*}\right), \mu^{*} \chi_{0.025}^{2}\left(2 a^{*}\right) /\left(2 a^{*}\right)\right),
$$

where $\chi_{\alpha}^{2}(d f)$ is the value for the chi-squared distribution with $d f$ degrees of freedom that provides a probability of $\alpha$ to the right of the $\chi_{\alpha}^{2}(d f)$ value.

Next, we turn to statistical inference for the parameter of effective vehicle length $L_{e}$. The posterior distribution of $L_{e}$ is

$$
p\left(L_{e} \mid x, y\right) \propto p\left(y \mid x, L_{e}\right) p\left(L_{e}\right)=\sum_{i=1}^{Q} q_{i} p\left(y \mid x, L_{e}\right) g\left(L_{e} ; b_{i}, b_{i} \lambda_{i}^{-1}\right) .
$$

Note that

$$
p\left(y \mid x, L_{e}\right) g\left(L_{e} ; b_{i}, b_{i} \lambda_{i}^{-1}\right)=B_{i} g\left(L_{e} ; \beta+b_{i},(\beta-1)(M y / x)^{-1}+b_{i} \lambda_{i}^{-1}\right),
$$


where $B_{i}=\left\{\Gamma\left(\beta+b_{i}\right) / \Gamma\left(b_{i}\right)\right\}\left\{b_{i} / \lambda_{i}\right\}^{b_{i}} /\left\{(\beta-1)(M y / x)^{-1}+b_{i} \lambda_{i}^{-1}\right\}^{\beta+b_{i}}$ is a constant. Hence, letting $\left.\quad \lambda_{i}^{*-1}=\left\{(\beta-1)(M y / x)^{-1}+b_{i} \lambda_{i}^{-1}\right)\right\} /\left(\beta+b_{i}\right), \quad b_{i}^{*}=\beta+b_{i}, \quad$ and $\quad q_{i}^{*}=q_{i} B_{i} / B_{0} \quad$ with $B_{0}=\sum_{i=1}^{Q} q_{i} B_{i}$, we obtain

$$
p\left(L_{e} \mid x, y\right)=\sum_{i=1}^{Q} q_{i}^{*} g\left(L_{e} ; b_{i}^{*}, b_{i}^{*} \lambda_{i}^{*-1}\right)
$$

Clearly the posterior distribution of $L_{e}$ retains the same functional form as the prior distribution (4-1) with the parameters updated.

Now we focus on each vehicle class characterized by the component $g\left(L_{e} ; b_{i}^{*}, b_{i}^{*} \lambda_{i}^{*-1}\right)$ of the posterior density. For vehicles falling into class $i$, the posterior mean and posterior mode of $L_{e}$ are:

$$
\begin{aligned}
& E\left(L_{e} \mid x, y, \text { vehicle class }=i\right)=\lambda_{i}^{*}=\left\{\omega_{i} \lambda_{i}^{-1}+\left(1-\omega_{i}\right) c D^{-1}\right\}^{-1}, \\
& \operatorname{mode}\left(L_{e} \mid x, y, \text { vehicle class }=i\right)=\lambda_{i}^{*}\left(b_{i}^{*}-1\right) / b_{i}^{*},
\end{aligned}
$$

where weight $\omega_{i}$ is given by $\omega_{i}=b_{i} /\left(\beta+b_{i}\right) . \quad c=(\beta-1) / \beta . D=s y$ is the calculated effective vehicle length using the classical method in equation (2-2). Note that $\lambda_{i}^{*}$ is a weighted harmonic average of the prior estimate and the current observation. Clearly when $b_{i}^{*}$ is large, the posterior mean and posterior mode are very close to each other.

Similarly, for vehicles falling into the class $i$, the posterior variance of $L_{e}$ is

$$
\operatorname{var}\left(L_{e} \mid x, y, \text { vehicle class }=i\right)=\lambda_{i}^{* 2} / b_{i}^{*} .
$$

Finally, from equations (4-7) and (4-8), we obtain the posterior mean and the posterior variance of $L_{e}$ as follows:

$$
E\left(L_{e} \mid x, y\right)=\sum_{i=1}^{Q} q_{i}^{*} \lambda_{i}^{*} \quad \text { and } \quad \operatorname{var}\left(L_{e} \mid x, y\right)=\sum_{i=1}^{Q} q_{i}^{*} \lambda_{i}^{* 2}\left(1+1 / b_{i}^{*}\right)-\left\{\sum_{i=1}^{Q} q_{i}^{*} \lambda_{i}^{*}\right\}^{2}
$$




\subsection{Predictive distribution and vehicle classification}

On the basis of the posterior distribution of $L_{e}$, the posterior predictive distribution of $L_{e}$ can be derived.

Theorem 2. Let $\tilde{x}$ and $\tilde{y}$ be the times required by a vehicle to traverse the dual-loop detector which follow observational distributions (3-1) and (3-2) respectively. Then conditional on $\tilde{x}$, the predictive distribution of $\tilde{r}=\tilde{y} / \tilde{x}$ has a mixture of $Q$ scaled $F$ distributions, each with degrees of freedom $2 b_{i}^{*}$ and $2 \beta$ respectively:

$$
p(\tilde{r} \mid \tilde{x})=\sum_{i=1}^{Q} q_{i}^{*} f_{i}(\tilde{r})
$$

where $\quad f\left(\tilde{r}_{i}\right)=A_{i} \tilde{r}^{b_{i}^{*}-1} /\left\{(\beta-1) / M+b_{i}^{*} \tilde{r} / \lambda_{i}^{*}\right\}^{\beta+b_{i}^{*}}$ with a constant of $A_{i}=\left\{\Gamma\left(\beta+b_{i}^{*}\right) /\left(\Gamma(\beta) \Gamma\left(b_{i}^{*}\right)\right)\right\}\left\{b_{i}^{*} / \lambda_{i}^{*}\right\}^{b_{i}^{*}}\{(\beta-1) / M\}^{\beta}$.

In traffic engineering, vehicles are usually classified into several categories such as passenger cars and other smaller vehicles, vans and small lorries, large lorries and road trains (see, e.g., Nihan et al., 2006). A direct application of the predictive distribution of $L_{e}$ is vehicle classification. According to Bayes' rule, the posterior classification probability that the vehicle with a ratio of $\tilde{r}$ is from class $i$ can be calculated as follows:

$$
\operatorname{Pr}\{\text { vehicle } \in \text { class } i \mid \tilde{r}\}=q_{i}^{*} f_{i}(\tilde{r}) / \sum_{j=1}^{Q} q_{j}^{*} f_{j}(\tilde{r}) \quad(i=1, \ldots, Q)
$$

Hence, the vehicle with $\tilde{r}=\tilde{y} / \tilde{x}$ is classified into a class, say $i *$ if $\operatorname{Pr}\left\{\right.$ vehicle $\in$ class $\left.i^{*} \mid \tilde{r}\right\}$ has the largest posterior classification probability. See Press (2003, Chapter 16) and Everitt et al. (2001, Chapter 6) for details. 


\subsection{An algorithm}

Comparing the prior distribution of $v$ specified in (4-2) with the posterior distribution of $v$ in (4-5), we can see that the same functional form is retained after applying Bayes' rule. Hence the prior of $v$ is a natural conjugate prior distribution. An important implication is that when the next piece of information is available, the obtained posterior distribution of the speed parameter $v$ in equation (4-5) can be treated as a prior distribution, and Bayesian analysis can be performed in the manner outlined in the previous subsection.

In addition, we note that vehicle speed evolves slowly over time in practice. To take this into account, we follow Li's (2009) approach and define a 'forgetting' factor so that observations collected at different times are weighted differently when they are used to estimate vehicle speed, with the latest observations given the largest weights. Specifically, instead of equation (4-2), the prior distribution is now specified as

$$
v \sim \Gamma\left(\delta_{v} a, \delta_{v} a \mu^{-1}\right),
$$

where $\delta_{v}$ is a forgetting factor lying in the interval $(0,1)$.

Although technically the same argument can be applied to the estimation of the effective vehicle length $L_{e}$, it does not make much sense in traffic engineering. In general, the length of one vehicle will provide little guidance as to the likely length of the following vehicle. For instance, even if the length of the previous vehicle was 65 feet, it would be expected that the following vehicle to be a short vehicle. Hence, we use a time-invariant prior on the vehicle lengths in this paper. Specifically, before the online estimation starts, we first obtain a prior distribution of $L_{e}$, equation (4-1), using historical data. Then the variance of each component distribution in (4-1) is inflated via a forgetting factor $\delta_{L} \in(0,1)$ :

$$
p\left(L_{e}\right)=\sum_{i=1}^{Q} q_{i} g\left(L_{e} ; \delta_{L} b_{i}, \delta_{L} b_{i} \lambda_{i}\right)
$$


Unlike the case of speed estimation, however, the same prior (4-9) is incorporated for every vehicle.

In Bayesian statistics, the posterior mean or posterior mode of a parameter is usually used as an estimate of the parameter. For the posterior distribution of effective vehicle length $L_{e}$ which is a mixture of distributions, however, the posterior mean is not a sensible estimate as the lengths of long vehicles will be greatly underestimated. Moreover, an exact closed-form solution for the posterior mode is difficult to obtain. Note, however, we may classify a vehicle into the a posteriori most probable class. Since the component density of each vehicle class is reasonably symmetric, the mode of the density can be well approximated by the corresponding mean. Therefore, in this paper, the estimate of $L_{e}$ is taken as the posterior mean of the vehicle class that the current vehicle belongs to.

This results in an algorithm for the estimation of vehicle speed and length:

Algorithm. Given: Forgetting factors $\delta_{v}$ and $\delta_{L}$; Diffusion parameters $\alpha$ and $\beta$; $c=(\beta-1) / \beta$; Initial parameters $a_{0}, \mu_{0}, b_{i}, \lambda_{i}$, and $q_{i}(i=1, \ldots, Q)$. Let $b_{i}=\delta_{L} b_{i}$. Calculate $H_{i}=\left\{\Gamma\left(\beta+b_{i}\right) / \Gamma\left(b_{i}\right)\right\}\left\{b_{i} / \lambda_{i}\right\}^{b_{i}}$ and $\omega_{i}=b_{i} /\left(\beta+b_{i}\right)(i=1, \ldots, Q)$;

For $k=1: K$

Step 1. Collect current observations $x_{k}$ and $y_{k}$;

Step 2. Calculate $s_{k}=M / x_{k}, D_{k}=s_{k} y_{k}$, and $r_{k}=y_{k} / x_{k}$;

Step 3.1. Calculate $\left.B_{i}=H_{i} /\left\{(\beta-1) / D_{k}+b_{i} / \lambda_{i}\right\}^{\beta+b_{i}}\right\}, \quad B_{0}=\sum_{i=1}^{Q} q_{i} B_{i} \quad$ and $\quad q_{i}^{*}=q_{i} B_{i} / B_{0}$ $(i=1, \ldots, Q)$

Step 3.2. Calculate the posterior classification probability: 
$\operatorname{Pr}\left\{\right.$ vehicle $\in$ class $\left.i \mid r_{k}\right\}=q_{i}^{*} f_{i}\left(r_{k}\right) / \sum_{j=1}^{Q} q_{j}^{*} f_{j}\left(r_{k}\right) \quad(i=1, \ldots, Q)$.

Classify the vehicle into class $i^{*}$ such that $\operatorname{Pr}\left\{\right.$ vehicle $\in$ class $\left.i^{*} \mid r_{k}\right\}$ is the largest.

Step 3.3. Estimate $L_{e}$ by $\lambda_{i^{*}}=\left\{\omega_{i^{*}} \lambda_{i^{*}}^{-1}+\left(1-\omega_{i^{*}}\right) c D_{k}^{-1}\right\}^{-1}$;

Step 4. Calculate weight $\theta_{k}=a_{k} /\left(a_{k}+\alpha\right)$, estimate $v$ by $\mu_{k}=\left\{\theta_{k} \mu_{k-1}^{-1}+\left(1-\theta_{k}\right) s_{k}^{-1}\right\}^{-1}$, and update the shape parameter as $a_{k}=\delta_{v}\left(a_{k-1}+\alpha\right)$;

End.

The forgetting factors $\delta_{v}$ and $\delta_{L}$ are usually treated as tuning parameters and are determined experimentally. A suitable choice for the forgetting factors depends on the variability of traffic flow and the quality of the priors. For instance, a smaller value of the forgetting factor $\delta_{v}$ is preferable for a larger variability in the speed evolution because the information on vehicle speed obtained previously becomes less relevant in this situation. Likewise, a larger value of the forgetting factor $\delta_{L}$ is preferable for a higher quality of the prior for effective vehicle length.

In addition, it is worth noting that when the chosen factors $\delta_{v}$ and $\delta_{L}$ are sufficiently small, weights $\theta_{k}$ and $\omega_{i}$ will be close to zero. Consequently the estimated vehicle speed and effective vehicle length reduce to those obtained using the classical method given in equations (2-1) and (2-2). Therefore the developed method includes the classical method as its special case.

\subsection{Estimation of the diffusion parameters}


The diffusion parameters $\alpha$ and $\beta$ used in the foregoing algorithm need to be estimated using historical data.

First we consider the diffusion parameter $\alpha$. From the marginal distribution of $x$ in (3-1), the mean and variance of $x$ are equal to $E_{x}=M / v$ and $V_{x}=(M / v)^{2} / \alpha$ respectively. Hence, we obtain $\alpha=\left(E_{x}\right)^{2} / V_{x}$.

Next, from Theorem 1, the mean and variance of $y$ are given by $E_{y}=L_{e} / v$ and $V_{y}=\left(L_{e} / v\right)^{2} /\left(\alpha \rho^{2}\right)$ respectively. Combining the two equations we have $\rho=\left(E_{y}\right)^{2} /\left(\alpha V_{y}\right)$. By recalling $\rho=\{(\beta-2) /(\alpha+\beta-1)\}^{1 / 2}$, we obtain $\beta=((\alpha-1) \rho+2) /(1-\rho)$.

The diffusion parameters $\alpha$ and $\beta$ can thus be estimated using the method of moments where the theoretical means and variances of $x$ and $y$ in the above equations are replaced with their sample counterparts.

\subsection{Information pooling}

For simplicity, the statistical analysis carried out so far has been based on the definition of $x=t_{d o n}-t_{u o n}$ and $y=t_{d o f f}-t_{d o n}$ (which are denoted as $x_{1}$ and $y_{1}$ respectively in this subsection). In practice however, when the two loops have sensitivity regions of equal size, one may choose $x_{2}=t_{\text {doff }}-t_{\text {uoff }}$ and $y_{2}=t_{\text {uoff }}-t_{\text {uon }}$ instead, and then perform statistical inference using the paired data $\left(x_{2}, y_{2}\right)$ rather than $\left(x_{1}, y_{1}\right)$. This will produce a slightly different numerical result because $\left(x_{2}, y_{2}\right)$ and $\left(x_{1}, y_{1}\right)$ may not be exactly the same.

To solve this problem, the classical method uses a modified version of equations (2-1) and (2-2) and incorporates the following pooled estimates of vehicle speed and effective vehicle length (see, e.g., Zhang et al. 2006):

$$
\hat{s}=\left\{s_{1}+s_{2}\right\} / 2 \text { and } \hat{D}=\left\{\hat{s} y_{1}+\hat{s} y_{2}\right\} / 2
$$


where $s_{1}=M / x_{1}$ and $s_{2}=M / x_{2}$.

Similarly, we may also pool the two data pairs $\left(x_{1}, y_{1}\right)$ and $\left(x_{2}, y_{2}\right)$ in the Bayesian analysis. Specifically, for each vehicle $k$ passing through the detector and for the prior (4-3) obtained from vehicle $k-1$, we first perform the Bayesian analysis using the data pair $\left(x_{1}, y_{1}\right)$. Then we treat the obtained posterior as the prior without inflating its variance, and perform the Bayesian analysis once again using the data pair $\left(x_{2}, y_{2}\right)$. From equations (4-4) and (47a), this results in the following estimates of the speed and effective vehicle length for vehicle $k$ :

$$
\mu_{k}=\left\{\theta_{k} \mu_{k-1}^{-1}+\left(1-\theta_{k}\right)\left(s_{1 k}^{-1}+s_{2 k}^{-1}\right) / 2\right\}^{-1},
$$

and $\quad \lambda_{i^{*}}^{*}=\left\{\omega_{i^{*}} \lambda_{i^{*}}^{-1}+\left(1-\omega_{i^{*}}\right) c\left(D_{1 k}^{-1}+D_{2 k}^{-1}\right) / 2\right\}^{-1}$,

where the weights are calculated as $\theta_{k}=a_{k} /\left(a_{k}+2 \alpha\right)$ and $\omega_{i}=b_{i} /\left(b_{i}+2 \beta\right) \quad(i=1, \ldots, Q)$ respectively. The measurements on vehicle speed and effective vehicle length are given by $s_{1 k}=M / x_{1 k}, s_{2 k}=M / x_{2 k}, D_{1 k}=s_{1 k} y_{1 k}$ and $D_{2 k}=s_{2 k} y_{2 k}$. Note that here the subscript $k$ is used for the time measurements $x_{1}, y_{1}, x_{2}$ and $y_{2}$ on vehicle $k$.

There are a couple of things worth noting here. First, the recursive formulas (4-11) and (4-12) indicate that if $\hat{s}_{k}=2\left(s_{1 k}^{-1}+s_{2 k}^{-1}\right)^{-1}$ and $\hat{D}_{k}=2\left(D_{1 k}^{-1}+D_{2 k}^{-1}\right)^{-1}$ are treated as combined measurements on vehicle speed and effective vehicle length, the foregoing algorithm can still be used.

Next, it is easy to verify that the obtained posterior for vehicle $k$ does not depend on the order of the Bayesian analyses, i.e. the first Bayesian analysis can be performed using either $\left(x_{1}, y_{1}\right)$ or $\left(x_{2}, y_{2}\right)$, and the remaining paired data are used in the second Bayesian analysis.

In addition, compared to the classical method, we can see the arithmetic average of the two measurements on speed (or effective vehicle length) in equation (4-10) is replaced by the 
corresponding harmonic average $\hat{s}_{k}=2\left(s_{1 k}^{-1}+s_{2 k}^{-1}\right)^{-1}\left(\right.$ or $\left.\hat{D}_{k}=2\left(D_{1 k}^{-1}+D_{2 k}^{-1}\right)^{-1}\right)$ in the Bayesian analysis.

Finally, we note that the paired data $\left(x_{1}, y_{1}\right)$ are in fact correlated with $\left(x_{2}, y_{2}\right)$. For instance, both $x_{1}$ and $y_{2}$ (or $x_{2}$ and $y_{1}$ ) by definition include a common time period of $t_{\text {uoff }}-t_{\text {uon }}$ (or $\left.t_{\text {doff }}-t_{d o n}\right)$. Consequently, the combined measurements on speed and effective vehicle length $\hat{s}_{k}=2\left(s_{1 k}^{-1}+s_{2 k}^{-1}\right)^{-1}$ and $\hat{D}_{k}=2\left(D_{1 k}^{-1}+D_{2 k}^{-1}\right)^{-1}$ are unduly over-weighed in the weights of $\theta_{k}=a_{k} /\left(a_{k}+2 \alpha\right)$ and $\omega_{i}=b_{i} /\left(b_{i}+2 \beta\right)(i=1, \ldots, Q)$. To adjust for this, a discount factor, $\Delta(1<\Delta<2)$, is required:

$$
\theta_{k}=a_{k} /\left(a_{k}+\Delta \alpha\right)=\left(a_{k} / \Delta\right) /\left[\left(a_{k} / \Delta\right)+\alpha\right]
$$

and $\quad \omega_{i}=b_{i} /\left(b_{i}+\Delta \beta\right)=\left(b_{i} / \Delta\right) /\left[\left(b_{i} / \Delta\right)+\beta\right] \quad(i=1, \ldots, Q)$.

Clearly, the discount factor $\Delta$ can be absorbed into the forgetting factors by defining $\tilde{\delta}_{v}=\delta_{v} / \Delta, \tilde{\delta}_{L}=\delta_{L} / \Delta, a_{k}=\tilde{\delta}_{v}\left(a_{k-1}+\alpha\right)$ and $b_{i}=\tilde{\delta}_{L} b_{i}$ in the foregoing algorithm. Hence it does not incur any extra computational cost.

\section{Numerical examples}

In this section two numerical examples illustrate the developed method.

\subsection{A simulation study}

Consider a dual-loop detector that consists of two identical single loops. Both loops are 6 feet long, and the distance between the two single loops is 16 feet. For simplicity it is assumed that the two loops have the same sensitivity.

\subsubsection{Data simulation}


Vehicle speed was assumed to evolve slowly over time and the 'true' values of the vehicle speed were simulated using random walks having an initial value of $60 \mathrm{mph}$ and a standard deviation of $1 \mathrm{mph}$. Only those speed realizations under $100 \mathrm{mph}$ were considered.

The 'true' distribution of the effective vehicle length was set as a mixture of three gamma distributions:

$$
\pi\left(L_{e}\right)=\sum_{i=1}^{3} q_{i} g\left(L_{e} ; b_{i}, b_{i} \eta_{i}^{-1}\right)
$$

with weights $q_{1}=0.80, q_{2}=0.15$, and $q_{3}=0.05$. The gamma components have mean vehicle lengths equal to $\eta_{1}=20, \eta_{2}=40$ and $\eta_{3}=75$ feet respectively with the shape parameters $b_{i}=150 \quad(i=1,2,3)$. The effective vehicle lengths were simulated from the 'true' distribution in equation (5-1).

The times required by a vehicle to traverse the distances of $M$ and $L_{e}$ were simulated as the outcomes of gamma random variables having distributions given in equations (3-1) and (3-2) with the specified values of $\alpha$ and $\beta$ which characterize variability of the time measurements.

In each experiment, 500 vehicles were generated.

\subsubsection{Data analysis}

To apply the algorithm in Section 4.4 to analyze the data, the hyper-parameters in the initial prior of speed were specified as $a_{0}=0$ and $\mu_{0}=50$. The prior of effective vehicle length was set as $p\left(L_{e}\right)=\sum_{i=1}^{3} q_{i} g\left(L_{e} ; b_{i}, b_{i} \lambda_{0 i}^{-1}\right)$, where the prior mean vehicle length of each vehicle class was taken as $\lambda_{0 i}=(1+0.05) \eta_{i}$, i.e. the prior means have $5 \%$ errors compared to the 'true' mean vehicle lengths in (5-1). For simplicity, we set $\delta=\delta_{v}=\delta_{L}$. Two values of the common forgetting factor $\delta$ were considered in the experiments, 0.95 and 0.90 . 
To assess the accuracy of estimation, root mean squared errors (RMSEs) between the 'true' and estimated values of the vehicle speed, and between the 'true' and estimated values of the effective vehicle length, were calculated. The same data were also analyzed using the classical method. For each choice of the forgetting factor $\delta$ and the shape parameters $\alpha$ and $\beta$, in total 100 experiments were carried out and the average values of RMSEs over the 100 experiments are displayed in Table 1.

(Table 1 is about here)

It is clear from table 1 that the new method outperformed the classical method. In theory, this is due to the fact that the new method uses more information for statistical inference. Technically, the new method includes the classical method as its special case. Therefore by tuning the two forgetting factors, the new method can always have a performance which is not worse than that of the classical method.

In addition, we can see from Table 1 that using the new method is more beneficial for the estimation of vehicle speed. This is not surprising because up-to-date prior knowledge on vehicle speed was used in the foregoing algorithm to estimate the speed parameter. In contrast, a fixed prior distribution was incorporated for the effective vehicle length.

\subsection{A practical example}

We next consider a real traffic-flow example. The data used in the analysis were collected between 10:04 a.m. and 11:06 a.m. on a weekday in December 2004 by the Advanced Loop Event Data Analyzer (ALEDA) system at a dual-loop station in lane 1; see Nihan et al. (2005) for the details of the ALEDA system. This dual-loop detector consists of two single loops, each 6 feet long, separated by a distance of 16 feet. The traffic controller located in the 
roadside control cabinet scanned the dual-loop detector 64 times per second, resulting in a "loop occupied" or "loop not occupied" response. These signals were then converted into a sequence of vectors of observations, $T=\left[t_{\text {uon }}, t_{\text {uoff }}, t_{d o n}, t_{d o f f}\right]^{T}$. It is assumed that the two loops have the same sensitivity. The data was pre-screened using the algorithm developed by Zhang et al. (2006). The Bayesian analysis was performed using both the data pairs $\left(x_{1}, y_{1}\right)$ and $\left(x_{2}, y_{2}\right)$ as discussed in Section 4.6 where the estimates of the vehicle speed and effective vehicle length were updated via equations (4-11) and (4-12).

For simplicity, the two forgetting factors were set as $\tilde{\delta}_{v}=\widetilde{\delta}_{L}=0.9$. A vague initial prior of $v$ with hyper-parameters $a_{0}=0$ and $\mu_{0}=50 \mathrm{mph}$ was used in the analysis. A prior distribution of $L_{e}$ was obtained using effective vehicle length data. It turned out that a mixture of three gamma distributions fitted the length data well, as displayed in Figure 2. The three component distributions have means of $20.65,32.49$, and 74.28 feet respectively. This is in line with the empirical results in Hazelton (2004). Not surprisingly, majority of vehicles were small cars. The other two vehicle classes may be interpreted as vans and small lorries, and large lorries and road trains.

(Figure 2 is about here)

Figure 2. The prior distribution of effective vehicle length characterized by a mixture of three gamma distributions.

The estimated vehicle speed (real line) with the envelope of a nominal 95\% credible interval (the dotted lines) is displayed in Figure 3. It can be seen that the vehicle speed was at the level of about $56 \mathrm{mph}$ during the time period of interest. The nominal $95 \%$ credible interval was narrow and the average margin of error was $\pm 4.0 \mathrm{mph}$. 
(Figure 3 is about here)

Figure 3. The estimates of the vehicle speed using the developed method (real line) and the associated envelop of a nominal 95\% credible intervals (dotted lines).

The estimated effective vehicle length is displayed in Figures 4. It can be seen that there were a considerable number of large lorries travelling in this lane (lane 1). The method of vehicle classification developed in this paper classified $85 \%$ of the vehicles into the category of small cars, $11 \%$ into the category of vans and small lorries, and $4 \%$ into the category of large lorries and road trains.

(Figure 4 is about here)

Figure 4. The estimates of the effective vehicle length using the developed method.

\section{Concluding remarks}

A new method has been developed to draw statistical inference for vehicle speed and length using dual-loop data. The resulting formulas have nice analytical forms, where both the estimates of vehicle speed and vehicle length are updated as a weighted harmonic average of the previous estimate and the currently collected observation. The proposed method includes the classical estimation method as a special case where the forgetting factors are chosen to be small. As a by-product, vehicle classification is also investigated using Bayesian posterior classification probabilities. From a practical point of view, the developed method incurs a minimum computational cost and therefore the extra computing power needed in the controllers of dual-loop detectors is limited. 


\section{Acknowledgements}

The author would like to thank the referee for his/her constructive comments on earlier versions of this paper, and to thank Dr Patikhom Cheevarunothai, Professor Yinhai Wang, and Professor Nancy Nihan in the Department of Civil \& Environmental Engineering, University of Washington, for kindly providing the real traffic data analyzed in this paper.

\section{Appendix. Proofs of theorems}

Proof of Theorem 1. From equation (3-4), the marginal distribution of $y$ is given by

$$
\begin{aligned}
& p\left(y \mid v, L_{e}\right)=\int p\left(x, y \mid v, L_{e}\right) d x \\
& =c_{1} y^{-(\beta+1)} \int x^{\alpha+\beta-1} \exp \left\{-\left[\alpha v / M+(\beta-1) L_{e} /(M y)\right] x\right\} d x
\end{aligned}
$$

Since

$$
\int x^{\alpha+\beta-1} \exp \left\{-\left[\alpha v / M+(\beta-1) L_{e} /(M y)\right] x\right\} d x=\Gamma(\alpha+\beta)\left[\alpha \nu / M+(\beta-1) L_{e} /(M y)\right]^{-(\alpha+\beta)},
$$

we obtain

$$
p\left(y \mid v, L_{e}\right)=c_{2} y^{\alpha-1} /\left[(\beta-1) L_{e}+\alpha v y\right]^{(\alpha+\beta)},
$$

where $c_{2}=(\alpha v)^{\alpha}\left\{(\beta-1) L_{e}\right\}^{\beta} \Gamma(\alpha+\beta)\{\Gamma(\alpha) \Gamma(\beta)\}^{-1}$ is a constant. Hence, the marginal distribution of $y$ is a scaled $F$-distribution with degrees of freedom $2 \alpha$ and $2 \beta$ respectively.

Next, noting $E(x)=M / v$ and $E(y \mid x)=L_{e} x / M$, we have

$$
E(y)=E\{E(y \mid x)\}=E\left(L_{e} x / M\right)=L_{e} / v .
$$

Similarly, noting $\operatorname{var}(y \mid x)=\left(L_{e} x / M\right)^{2} /(\beta-2)$, we have

$$
\begin{aligned}
& \operatorname{var}(y)=E\{\operatorname{var}(y \mid x)\}+\operatorname{var}\{E(y \mid x)\}=E\left\{\left(L_{e} x / M\right)^{2} /(\beta-2)\right\}+\operatorname{var}\left\{L_{e} x / M\right\} \\
& =\operatorname{var}(x)\left(L_{e} / M\right)^{2}(\alpha+\beta-1) /(\beta-2) .
\end{aligned}
$$


Finally, from $E(x y)=E\{x E(y \mid x)\}=\left(L_{e} / M\right) E\left(x^{2}\right)$ and $E(x) E(y)=\left(L_{e} / M\right)\{E(x)\}^{2}$, we obtain $\operatorname{cov}(x, y)=E(x y)-E(x) E(y)=\left(L_{e} / M\right) \operatorname{var}(x)$. Then by combining these equations, we obtain the correlation coefficient $\rho=\{(\beta-2) /(\alpha+\beta-1)\}^{1 / 2}$.

Proof of Lemma 1. The proof for (i) is trivial. To show (ii), we note that the $r$ th moment of $g(z ; \phi, \phi)$ is $M_{1}=\phi^{-r} \Gamma(r+\phi) / \Gamma(\phi)$ with $\phi=\alpha \rho^{2}=\alpha(\beta-2) /(\alpha+\beta-1)$. On the other hand, the $r$ th moment of the distribution in (3-5) is equal to $M_{2}=\{(\beta-1) / \alpha\}^{r} \Gamma(\alpha+r) \Gamma(\beta-r) /\{\Gamma(\alpha) \Gamma(\beta)\}$. Hence by some algebra, we obtain

$$
M_{1} / M_{2}=\prod_{j=1}^{r}\{(\beta-j) /(\beta-2)\} \prod_{j=1}^{r}\{\alpha /(\alpha+r-j)\} \prod_{j=1}^{r}\{(\phi+r-j)(\alpha+\beta-1) /(\alpha(\beta-1)\} .
$$

Clearly, each of the above products is of order $1+O\left(\alpha^{-1}\right)+O\left(\beta^{-1}\right)$.

Proof of Theorem 2. By definition, the predictive distribution of $\tilde{r}$ for given $\tilde{x}$ is

$$
p(\tilde{r} \mid \tilde{x})=\int p\left(\tilde{r} \mid \tilde{x}, L_{e}\right) p\left(L_{e} \mid x, y\right) d L_{e}=\sum_{i=1}^{Q} q_{i}^{*} \int p\left(\tilde{r} \mid x, L_{e}\right) g\left(L_{e} ; b_{i}^{*}, b_{i}^{*} \lambda_{i}^{*-1}\right) d L_{e},
$$

where $p\left(\tilde{r} \mid \tilde{x}, L_{e}\right)$ is given by equation (3-3) and $p\left(L_{e} \mid x, y\right)$ is given by equation (4-6). Then it follows by noting that

$$
\int p\left(\tilde{r} \mid \tilde{x}, L_{e}\right) g\left(L_{e} ; b_{i}^{*}, b_{i}^{*} \lambda_{i}^{*-1} d L_{e}\right) d L_{e}=A_{i} \tilde{r}^{b_{i}^{*}-1}\left[(\beta-1) / M+b_{i}^{*} \lambda_{i}^{*-1} \tilde{r}\right]^{\beta+b_{i}^{*}}
$$

is a scaled $F$-distribution having degrees of freedom $2 b_{i}^{*}$ and $2 \beta$ respectively with $A_{i}=\left\{\Gamma\left(\beta+b_{i}^{*}\right) /\left(\Gamma(\beta) \Gamma\left(b_{i}^{*}\right)\right)\right\}\left\{b_{i}^{*} / \lambda_{i}^{*}\right\}^{b_{i}^{*}}\{(\beta-1) / M\}^{\beta}$ a constant.

\section{References}

Bickel, P. J., Chen, C., Kwon, J., Rice, J., Van Zwet, E., Varaiya P., 2007. Measuring traffic. Statistical Science 22 (4), 581-597. 
Cheevarunothai, P., Wang, Y., Nihan, N. L., 2006. Identification and correction of dual-loop sensitivity problems. Transportation Research Record 1945, 73-81.

Coifman, B., Dhoorjaty, S., 2004. Event data-based traffic detector validation tests. Journal of transportation engineering 130 (3), 313-321.

Dailey, D. J., 1999. A statistical algorithm for estimating speed from single loop volume and occupancy measurements. Transportation Research part B 33 (5), 313-322.

Everitt, B. S., Landau, S., Leese, M., 2001. Cluster Analysis. Arnold, New York.

Hazelton, M., 2004. Estimating vehicle speed from traffic count and occupancy data. Journal of Data Science 2 (3), 231-244.

Kotz, S., Balakrishnan, N., Johnson, N. L., (2000). Continuous Multivariate Distributions. Vol. $1,2^{\text {nd }}$ ed. Wiley, New York.

Kurkjian, A., Gershwin, S., Houpt, P. Willsky, A., Chow, E., 1980. Estimation of roadway traffic density on freeways using presence detector data. Transportation Science 14 (3), 232-261.

Li, B., 2009. On the recursive estimation of vehicular speed using data from a single inductance loop detector: a Bayesian approach. Transportation Research Part B 43 (4), $391-402$.

McLachlan, G. J., 1996. The EM Algorithm and Extensions. Wiley, New York.

Nihan, N. L., Wang, Y., Cheevarunothai, P, 2006. Improving dual-loop truck (and speed) data: quick detection of malfunctioning loops and calculation of required adjustments. Technical Report WA-RD 647.1, Washington State Transportation Center, University of Washington.

Polus, A., 1979. A study of travel time and reliability on arterial routes. Transportation 8 (2), 141-151.

Press, S. J., 2003. Subjective and Objective Bayesian Statistics. Wiley, New York. 
Wang, Y., Nihan, N. L., 2000. Freeway traffic speed estimation with single-loop outputs. Transportation Research Record 1727, 120-126.

Ye, Z. R., Zhang, Y. L., Middleton, D. R., 2006. Unscented Kalman filter method for speed estimation using single loop detector data. Transportation Research Record 1968, 117125.

Zhang, X., Wang, Y., Nihan, N. L., 2006. Robust algorithm for improved dual-loop detection on freeways. Transportation Research Board Annual Meeting, Washington DC. 
Table 1

RMSEs of the estimates of effective vehicle length and vehicle speed using the new method and the classical method

\begin{tabular}{|c|c|c|c|c|c|c|}
\hline & & $\begin{array}{l}\text { Forgetting } \\
\text { factor }\end{array}$ & $\begin{array}{l}\text { New } \\
\text { method }\end{array}$ & $\begin{array}{l}\text { Classic } \\
\text { method }\end{array}$ & $\begin{array}{l}\text { New } \\
\text { method }\end{array}$ & $\begin{array}{l}\text { Classic } \\
\text { method }\end{array}$ \\
\hline \multirow{3}{*}{$\alpha=100$} & \multirow{3}{*}{$\beta=80$} & & \multicolumn{2}{|c|}{ Vehicle length (ft) } & \multicolumn{2}{|c|}{ Vehicle speed (mph) } \\
\hline & & $\delta=0.95$ & 2.57 & 3.32 & 3.14 & 6.11 \\
\hline & & $\delta=0.90$ & 2.56 & 3.31 & 2.55 & 6.21 \\
\hline \multirow[t]{2}{*}{$\alpha=80$} & $\beta=50$ & $\delta=0.95$ & 3.39 & 4.22 & 3.14 & 6.93 \\
\hline & & $\delta=0.90$ & 3.39 & 4.20 & 2.63 & 6.87 \\
\hline \multirow[t]{2}{*}{$\alpha=50$} & $\beta=50$ & $\delta=0.95$ & 3.39 & 4.17 & 3.27 & 8.82 \\
\hline & & $\delta=0.90$ & 3.36 & 4.17 & 2.88 & 8.82 \\
\hline
\end{tabular}




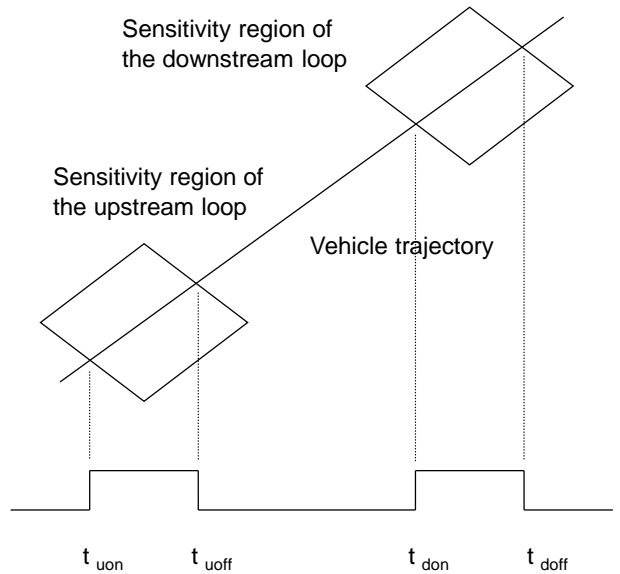

Figure 1. An illustration of a dual-loop detector. 


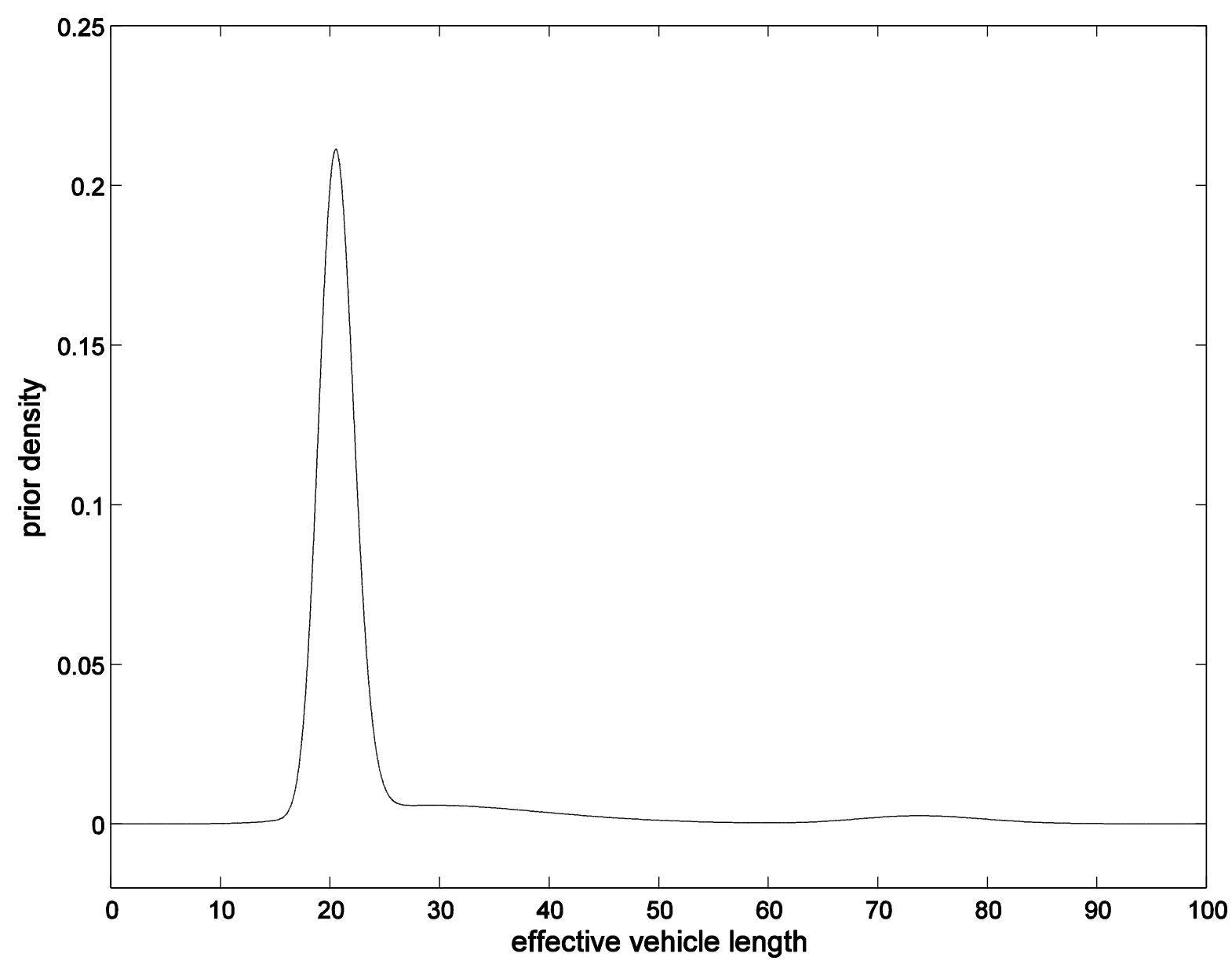

Figure 2. The prior distribution of effective vehicle length characterized by a mixture of three gamma distributions. 


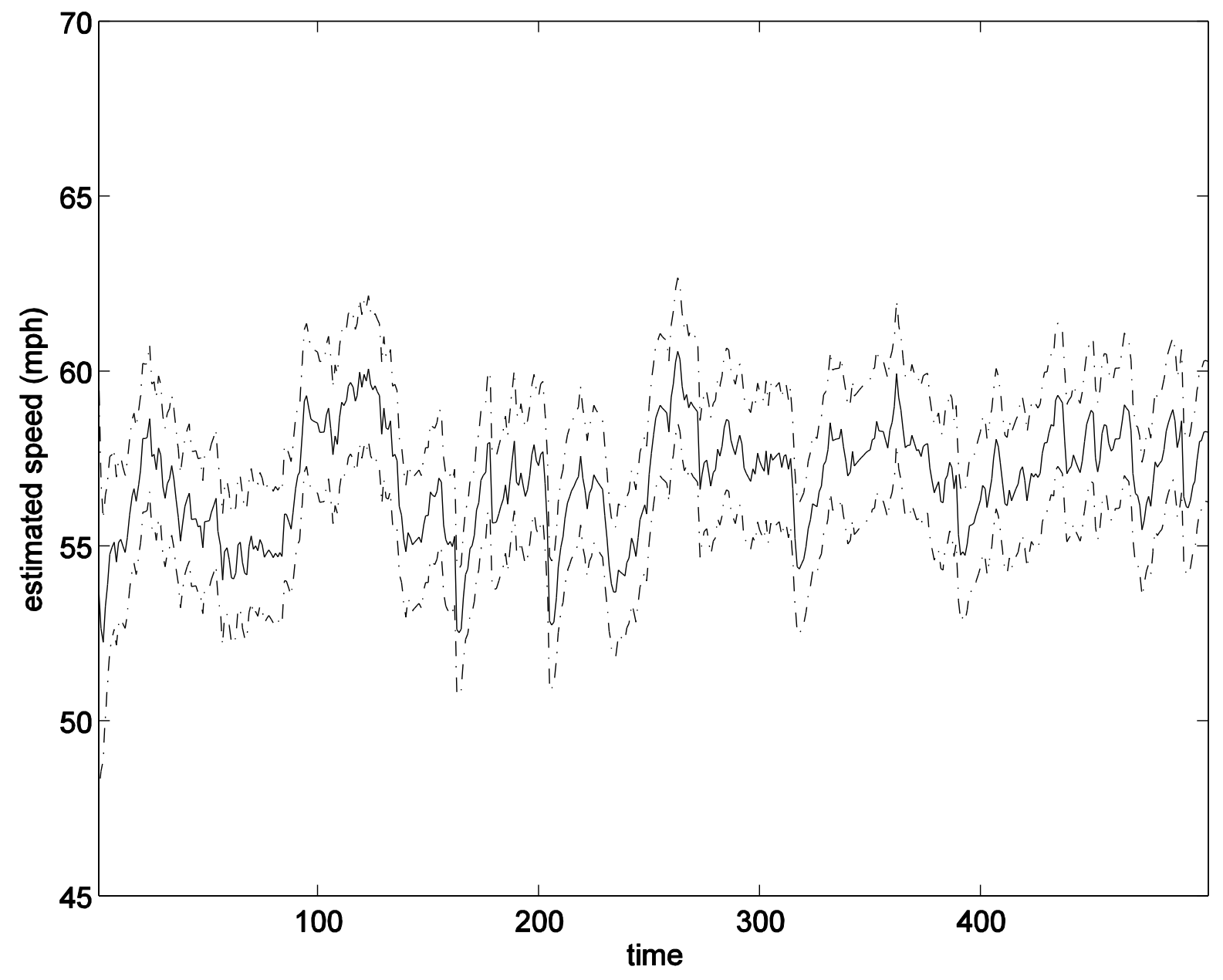

Figure 3. The estimates of the vehicle speed using the developed method (real line) and the associated envelop of a nominal 95\% credible intervals (dotted lines). 


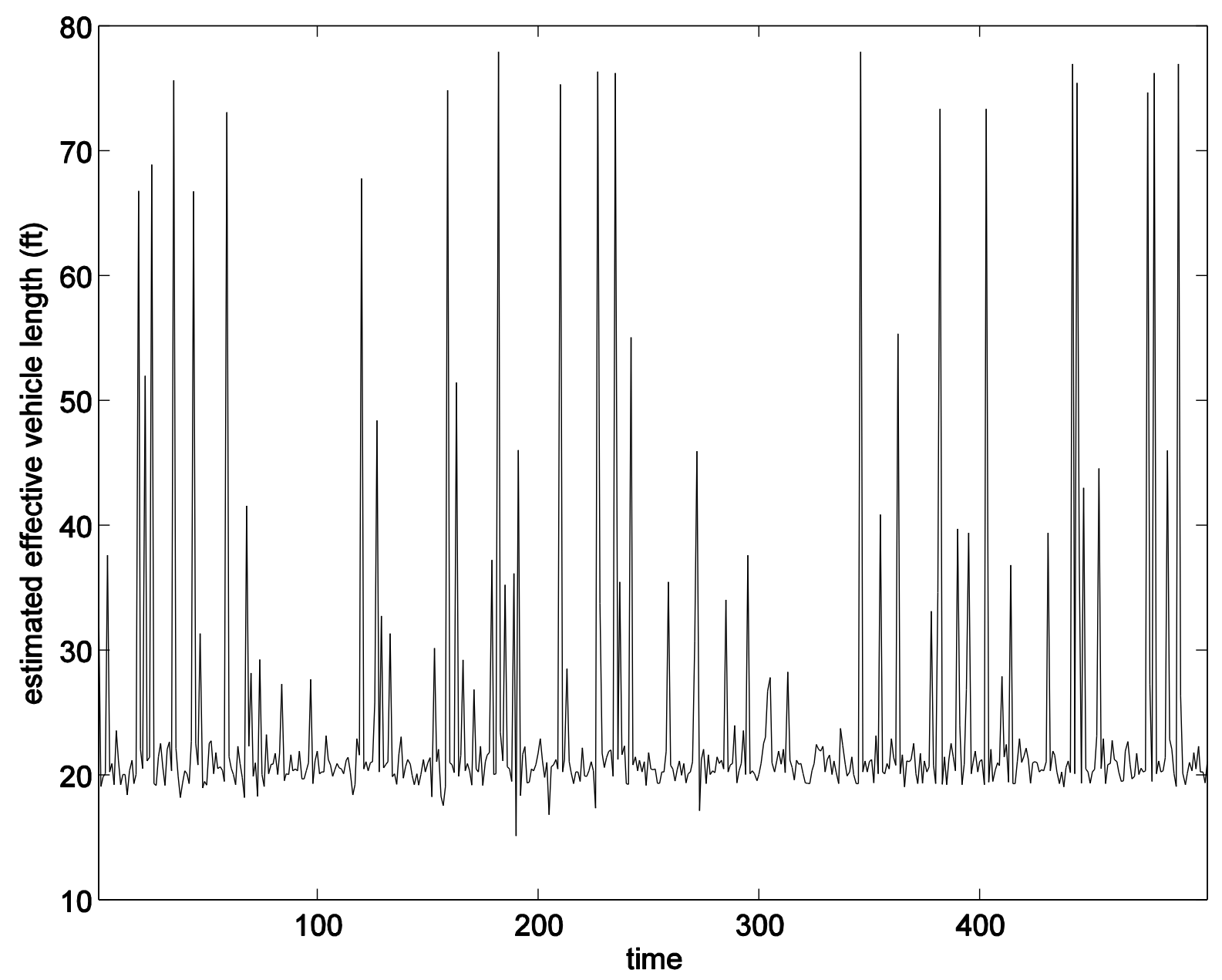

Figure 4. The estimates of the effective vehicle length using the developed method. 